\title{
PARTICLE DYNAMICS AT STAGNATION POINT DURING LONGITUDINAL BUNCH COMPRESSION OF HIGH CURRENT BEAMS*
}

\author{
T. Kikuchi ${ }^{\dagger}$, S. Kawata, Utsunomiya Univ., Utsunomiya 321-8585, Japan \\ K. Horioka, Tokyo Institute of Technology, Yokohama 226-8502, Japan
}

\begin{abstract}
Beam dynamics around a stagnation point during a longitudinal pulse compression is numerically investigated by using a three dimensional multi-particle code developed with a reduced electric field model. A deformation of the beam particle distribution in the longitudinal phase space is observed during the beam transport with the pulse compression. An rms transverse emittance growth is additionally increased along the beam transport due to the disruption in the longitudinal distribution of the beam particle.
\end{abstract}

\section{INTRODUCTION}

An intense heavy-ion beam (HIB) is one influential candidate as the energy driver, and the physics of spacecharge-dominated beams is crucial in heavy ion inertial fusion (HIF) researches [1]. Required parameter values of HIB are several GeV particle energy, $\sim 100 \mathrm{kA}$ total current, and $\sim 10$ ns short pulse duration [2]. The beam parameters are far from those of conventional particle accelerator system. Therefore the beam dynamics and control are important research issues in HIF.

Not only for HIF application, but also research fields on high energy density physics (HEDP) and warm dense matter (WDM) sciences [3] driven by HIB illuminations require the generation of a high-current HIB [4] .

At the final stage, the beam pulse must be longitudinally compressed from $\sim 100$ to $\sim 10 \mathrm{~ns}$. Induction voltage modulators, which have a precise waveform controllability, are useful devices for the bunch compression [5]. For an effective target implosion, we should transport and compress the bunch of HIB with a low emittance growth. For this reason, the final pulse compression and the final focusing are a key technology in the HIF driver system. In these regions, the intense HIB is in the space-charge-dominated state, and beam instabilities may occur during the beam transport.

In our previous studies [6,7], the beam dynamics was investigated by using a two dimensional multi-particle code including the longitudinal pulse compression model. In this study, we carry out numerical simulations by using a three dimensional particle code developed. The longitudinal and transverse beam parameter changes are discussed from the numerical simulation results during the pulse compression. Disruption of the longitudinal particle distribution is shown in the beam transport, and implies that an emittance growth

\footnotetext{
* Work supported by Japan Society for the Promotion of Science KAKENHI No.17740361.

† tkikuchi@cc.utsunomiya-u.ac.jp
}

05 Beam Dynamics and Electromagnetic Fields larger than one near center of the beam bunch and twodimensional sliced code result is caused due to the longitudinal particle motions.

\section{SIMULATION MODEL AND BEAM PARAMETERS}

The calculation code used, which is based on a particlein-cell (PIC) method [8], takes into account of a selfelectrostatic and an external applied magnetic fields, and can be described in a three dimensional Cartesian coordinate. The particle motions are calculated in the fully three dimensional space by neglecting a self-magnetic field.

The electric field created due to the space charge is calculated by using the Poisson equation,

$$
\nabla^{2} \phi=\frac{\partial^{2} \phi}{\partial x^{2}}+\frac{\partial^{2} \phi}{\partial y^{2}}+\frac{\partial^{2} \phi}{\partial z^{2}}=-\frac{\rho}{\epsilon_{0}},
$$

where $\phi$ is the electrostatic potential, $\rho$ is the charge density, and $\epsilon_{0}$ is the permittivity of free space. By solving Eq. (1), the electric field can be completely determined by $\boldsymbol{E}=-\boldsymbol{\nabla} \phi$, however the higher computational cost is unaffordable for a lot of parameter studies. Vorobiev and York proposed a sub-three-dimensional PIC method [9], and the approach is as follows. The three dimensional Poisson equation Eq. (1) can be replaced as

$$
\frac{\partial^{2} \phi}{\partial x^{2}}+\frac{\partial^{2} \phi}{\partial y^{2}}=-\rho^{\prime}
$$

where

$$
\rho^{\prime}=\frac{\rho}{\epsilon_{0}}-\frac{\partial E_{z}}{\partial z} .
$$

If Eq. (3) can be solved with including the transverse information, we can obtain the transverse electric field by solving the two dimensional Poisson equation as shown in Eq. (2).

Assuming the large aspect ratio to longitudinal and transverse directions for the beam bunch, we employ the simplified calculation model for the longitudinal electric field in this study. The transverse electric fields are normally calculated by $E_{x}=-\partial \phi / \partial x$ and $E_{y}=-\partial \phi / \partial y$, while by assuming the long wave approximation the longitudinal electric filed can be given as

$$
E_{z}=-\frac{g}{4 \pi \epsilon_{0} \gamma_{0}^{2}} \frac{d \lambda}{d z}
$$

where $\gamma_{0}$ is the relativistic factor at the central energy for the beam and $\lambda$ is the line charge density. For a spacecharge dominated regime, $g$ is the geometry factor defined 


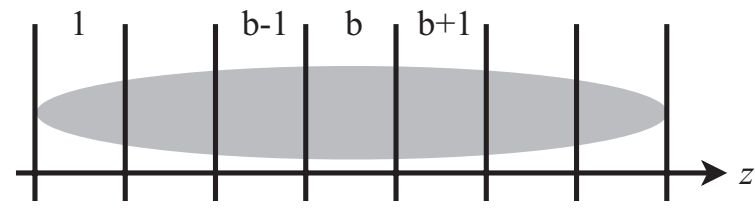

Figure 1: Sliced bunch model for the self-electric field calculations.

by

$$
g \sim \log \frac{r_{p}^{2}}{r_{x} r_{y}},
$$

where $r_{p}$ is the outer boundary pipe radius, $r_{x}$ and $r_{y}$ are effective beam radii estimated as

$$
r_{x}=2 \sqrt{\left\langle\left(x_{p}-\left\langle x_{p}\right\rangle\right)^{2}\right\rangle},
$$

with analogous expression for $r_{y}$. Here $x_{p}$ is the transverse position of the beam particle at the index $p$.

To reduce the computational cost, the beam bunch is longitudinally sliced and separated to calculate the transverse and longitudinal electric fields as shown in Fig. 1. At each time-step the bunches sliced are identified by using the index $b$. The transverse electric fields are calculated at each slice. For this reason, the horizontal and vertical electric fields are rewritten as $E_{x b}=-\partial \phi_{b} / \partial x$ and $E_{y b}=-\partial \phi_{b} / \partial y$ at each slice. Here the subscripts $b$ indicate the sliced bunch index. The electrostatic potential at each slice can be calculated by

$$
\frac{\partial^{2} \phi_{b}}{\partial x^{2}}+\frac{\partial^{2} \phi_{b}}{\partial y^{2}}=-\rho_{b}^{\prime}
$$

where

$$
\rho_{b}^{\prime}=\frac{\rho_{b}}{\epsilon_{0}}-\frac{d E_{z b}}{d z} .
$$

Here

$$
\frac{d E_{z b}}{d z}=-\frac{1}{4 \pi \epsilon_{0} \gamma_{0}^{2}} \frac{d}{d z}\left(g_{b} \frac{d \lambda_{b}}{d z}\right) .
$$

The Poisson equation at each slice can be numerically solved by using a multigrid and SOR methods [10].

The beam parameters are assumed as Table 1 [2]. The

Table 1: Beam parameters.

\begin{tabular}{cc}
\hline Ion species & $\mathrm{Pb}^{1+}$ \\
Number of ions & $6.25 \times 10^{14}$ \\
Particle energy [GeV] & 10 \\
Initial beam current [A] & 400 \\
Final beam current [kA] & 10 \\
Initial pulse duration [ns] & 250 \\
Final pulse duration [ns] & 10 \\
\hline
\end{tabular}

initial generalized perveance is assumed to be $3.58 \times 10^{-6}$. The initial undepressed and depressed phase advances are $\sigma_{0}=72 \mathrm{deg}$ and $\sigma=65.2 \mathrm{deg}$ at $3 \mathrm{~m}$ in the longitudinal direction. A continuous focusing (CF) configuration is assumed for the transverse confinement system. The transverse focusing coefficients $k_{x}=k_{y}=0.157 \mathrm{~m}^{-2}$ are constant for the CF model.

The transverse calculation region is fixed at the square of $10 \mathrm{~cm} \times 10 \mathrm{~cm}$, and the outer boundary condition is given as a conductor wall. As a result, the pipe radius $r_{p}$ is assumed as $5 \mathrm{~cm}$ in this study.

The rms matched Gaussian [11] beam is chosen as the initial particle (non-stationary) distribution for the transverse plane. The longitudinal charge distribution is assumed to be uniform before the bunch compression, on which a head-to-tail velocity tilt $\left(\beta_{t}-\beta_{h}\right) / \beta_{c}$ of $5 \%$ is applied. Here $\beta_{h}, \beta_{t}$, and $\beta_{c}$ are the velocity divided by light speed at the head, in the tail part and in the center of the beam, respectively.

\section{LONGITUDINAL AND TRANSVERSE BEAM PARAMETERS DURING PULSE COMPRESSION}

We simulate numerically the beam dynamics during the pulse compression with the $\mathrm{CF}$ focusing model as discussed in the previous section.

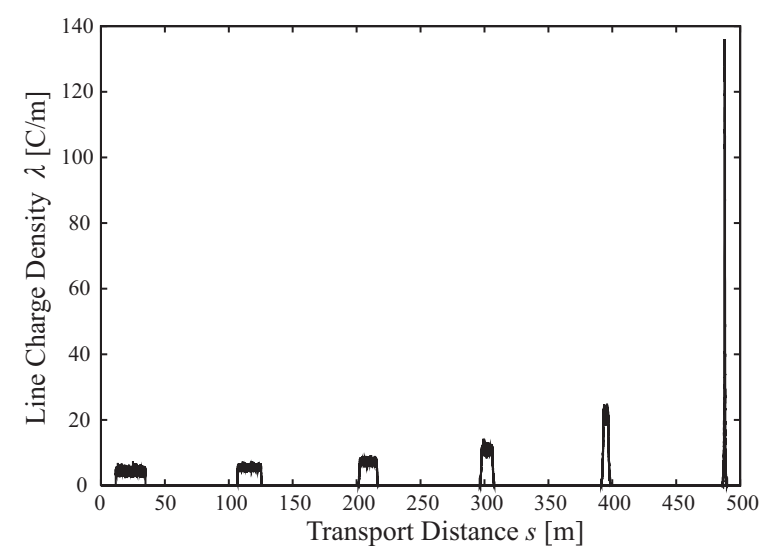

Figure 2: Line charge density along the beam transport direction.

Figure 2 shows the line charge density profiles at each longitudinal position during the beam transport. Due to the head-to-tail velocity tilt given initially, the beam bunch is longitudinally compressed during the transport, and the line charge density can be increased with the pulse compression.

The emittance value can be used to evaluate the beam quality. We define the average of unnormalized rms transverse emittance as $\varepsilon=\left(\varepsilon_{x, r m s}+\varepsilon_{y, r m s}\right) / 2$, where $\varepsilon_{x, r m s}$ and $\varepsilon_{y, r m s}$ are the unnormalized rms emittances for horizontal and vertical directions given by

$$
\varepsilon_{x, r m s}=\left[\left\langle x_{p}^{2}\right\rangle\left\langle x_{p}^{\prime 2}\right\rangle-\left\langle x_{p} x_{p}^{\prime}\right\rangle\right]^{1 / 2},
$$

and

$$
\varepsilon_{y, r m s}=\left[\left\langle y_{p}^{2}\right\rangle\left\langle y_{p}^{\prime 2}\right\rangle-\left\langle y_{p} y_{p}^{\prime}\right\rangle\right]^{1 / 2},
$$


where the prime (/) indicates the slope, i.e., $x_{p}^{\prime}=d x_{p} / d z_{p}$ and $y_{p}^{\prime}=d y_{p} / d z_{p}$, respectively. The initial emittance $\varepsilon_{i}$ is assumed to be $\varepsilon_{i}=10 \mathrm{~mm}$-mrad, and $\varepsilon_{x, r m s}=\varepsilon_{y, r m s}=$ $\varepsilon_{i}$. The evolutions of the emittance growth $\varepsilon / \varepsilon_{i}$, which indicates the ratio of the average emittance to the initial one along the transport distance, are shown in Fig. 3. The emittance growth over the entire beam is calculated by considering all beam particles, and the emittance growth near center is evaluated by the particles included in the range of $\pm 10 \mathrm{~cm}$ fixed from the longitudinal center of the beam bunch. As shown in Fig. 3, the emittance growth evaluated near center of the beam bunch is lower than one over the entire beam bunch. We also calculate the paticle dynamics in the transverse cross section of the beam bunch, and the effect of the longitudinal compression is performed as the current increase model. As shown in Fig. 2, the beam current is increased as $1 / z_{b}(s)$, where $z_{b}$ is the half bunch length at each transport distance $s$. For this reason, the beam current increase model is assumed to calculate the $2 \mathrm{D}$ sliced bunch. It is found that the 2D sliced calculation with the longitudinal compression model well represents the emittance growth history for the 3D calculation result.

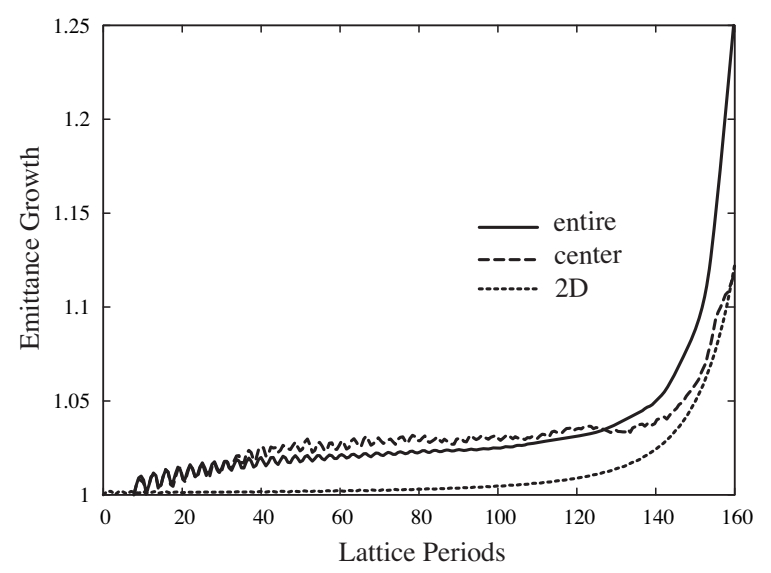

Figure 3: Evolution of the rms emittance during the beam transport with the pulse compression. The solid curve indicates the emittance growth over the entire beam bunch, the dashed line shows one evaluated near center of the beam bunch, and the dotted line shows for the 2D sliced calculation result with the longitudinal compression model.

Figure 4 shows the normalized charge distribution in the longitudinal phase space at the stagnation point. A disruption of the smooth particle distribution in the longitudinal phase space is indicated by Fig. 4 . The disruption is caused by increasing the amplitude of the longitudinal electric field due to the pulse compression. As a result, the large transverse emittance growth over the entire beam bunch can be caused due to the disruption of the particle distribution in the longitudinal phase space as shown in Fig. 4.

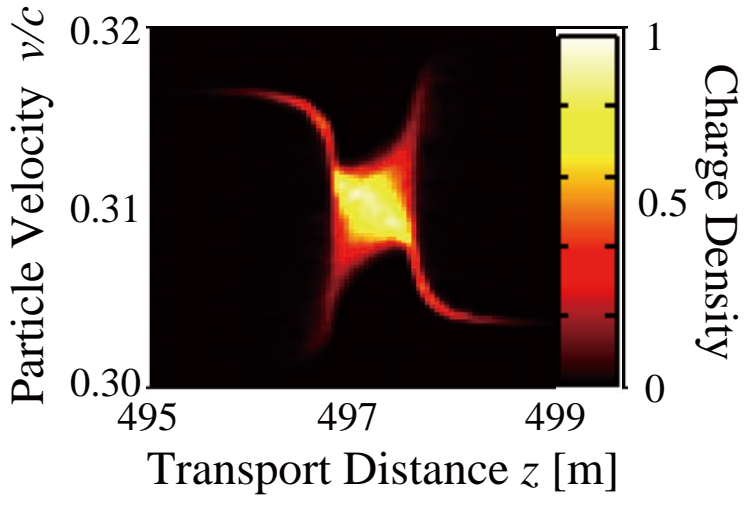

Figure 4: Normalized charge distribution in the longitudinal phase space at the stagnation point.

\section{CONCLUSIONS}

A high-current HIB dynamics during the beam transport with a longitudinal pulse compression was numerically investigated by using a three dimensional particle simulation code. The used code was developed to solve the three dimensional particle motions with the reduced electric field due to the space charge effect and the applied external magnetic field for the transverse confinement of the beam.

A disruption of smooth particle distribution in a longitudinal phase space during the pulse compression, which is caused due to the longitudinal self-electric field, was observed. A comparison of emittance growth between the all particles in the beam bunch, the particles included near center of the beam bunch and 2D sliced calculation result implies that the rms emittance growth over the entire beam can be increased due to the disruption of the longitudinal particle distribution.

\section{REFERENCES}

[1] J.J. Barnard, J. Fusion Energy 17, 223 (1998).

[2] J.J. Barnard, et al., Nucl. Instrum. Methods in Phys. Res. A415, 218 (1998).

[3] T. Sasaki, Y. Yano, M. Nakajima, T. Kawamura, K. Horioka, Nucl. Instrum. Methods in Phys. Res. A 577, 313 (2007).

[4] K. Horioka, et al., Nucl. Instrum. Methods in Phys. Res. A 577, 298 (2007).

[5] K. Horioka, et al., Laser Part. Beams 20, 609 (2002).

[6] T. Kikuchi, M. Nakajima, K. Horioka, and T. Katayama, Phys. Rev. ST Accel. Beams 7, 034201 (2004).

[7] T. Kikuchi, K. Horioka, M. Nakajima, S. Kawata, Nucl. Instrum. Methods in Phys. Res. A 577, 103 (2007).

[8] R.W. Hockney and J.W. Eastwood, Computer Simulation Using Particles, McGraw-Hill, New York, (1981).

[9] L.G. Vorobiev and R.C. York, Phys. Rev. ST Accel. Beams 3, 114201 (2000).

[10] U. Trottenberg, C.W. Oosterlee, and A. Schüller, MULTIGRID, Elsevier, San Diego, (2001).

[11] Y.K. Batygin, Nucl. Instrum. Methods in Phys. Res. A 539, 455 (2005).

05 Beam Dynamics and Electromagnetic Fields

D03 High Intensity - Incoherent Instabilities, Space Charge, Halos, Cooling 